\title{
Aggregating fuzzy $e$-Xor connectives based on the OWA-operator
}

\author{
Renata Hax Sander Reiser, \\ UFPEL, CDTec, PPGC \\ CP 354, 96001-970 Pelotas, RS, Brazil \\ E-mail: reiser@ucpel.tche.br, \\ Benjamín René Callejas Bedregal \\ UFRN, DIMAP, PPSC/PPgEEC \\ Campus Universitário, 1524, 59072-970, Natal, RN, Brazil \\ E-mail: bedregal@dimap.ufrn.br
}

\begin{abstract}
Abstrac: The main contribution of this paper is concerned with the aggregating fuzzy e-Xor connectives based on the OWA-operator focusing on the fuzzy e-Xor implication class and related dual construction. The paper formally states that such aggregation operator preserves the main properties of fuzzy connectives in corresponding classes.
\end{abstract}

Keywords: fuzzy logic, aggregation function, OWA-operator, fuzzy Xor, fuzzy Xor implications

\section{Introduction}

In Fuzzy Logic, the "fuzzy exclusive or" (Xor) and its dual construction - the XNor connective are extensively studied, e.g.[9, 8, 10,11] and [12], related to applications and basic concepts which are concerned with knowledge based systems, either from image processing to decision making or from pattern recognition to machine learning.

By aggregating a family of fuzzy $\mathrm{X}(\mathrm{N})$ or connectives making use of the ordered weighted average aggregation operator (OWA-operator), this paper deals with an extension of fuzzy $\mathrm{X}(\mathrm{N})$ or named as the fundamental class of representable fuzzy subX(N)or and corresponding fuzzy implications.

Triangular sub(co)norms and strong fuzzy negations are introduced in[12] introducing the aggregation of fuzzy $(\mathrm{S}, \mathrm{N})$ - and R-implications by the arithmetic media.

The results achieved in $[1,2]$ are here extended into a discussion on the fuzzy subXor class, mainly related to relax boundary conditions in the definition of the fuzzy Xor class. We also investigated under which conditions such class is preserved by $n$-ary aggregation functions.

Instances of the fuzzy subXor class are reported but they are restricted to the fuzzy $e$-subXor connectives, referred as $\mathrm{E}_{S, T}$. Thus, an $e$-Xor connective is obtained as a difference between a t-subconorm $S$ and a t-subnorm $T$. By taking the algebraic sum $S_{P}$ and its mutual dual construction, the product t-norm $T_{P}$, the main algebraic properties, dual construction and graphical representation of the $\mathrm{E}_{S_{P}, T_{P}}$ are discussed.

This paper presents the main results obtained from the OWA-operator performed over a finite family of $e$-subXor connectives, introducing the operator $\mathcal{E}_{\mathcal{A}}$, called $(\mathcal{A}, \mathcal{E})$-operator. Its dual construction $\mathcal{D}_{\mathcal{A}}$, called $(\mathcal{A}, \mathcal{D})$-operator is also considered including algebraic properties. Additionally, a new fuzzy sub(co)implication is obtained from the OWA-operator and a finite family in the fuzzy $e$-subX(N)or (co)implication class.

This paper is organized as follows. The next section describes the basic concepts of fuzzy connectives. In Section 3, the fuzzy $e$-Xor connective, dual construction and related $e$-Xor (co)implication class are discussed. As our main contribution, Section 4 shows the aggregating fuzzy $e-\mathrm{X}(\mathrm{N})$ or connectives and related (co)implications. Finally, conclusions are drawn in Section 5. 


\section{Preliminaries}

In this section, basic definitions related to the fuzzy connectives: fuzzy negation and triangular (co)norms (t-(co)norms) are considered. For that, let $U=[0,1]$ be the unit interval.

\subsection{Fuzzy Negations}

A function $N: U \rightarrow U$ is a fuzzy negation if

N1: $N(0)=1$ and $N(1)=0 ; \quad$ N2: If $x \geq y$ then $N(x) \leq N(y), \forall x, y \in U$.

If $N$ also verifies the involutive property, it is called a strong fuzzy negation (SFN) [6]:

N3: $N(N(x))=x, \forall x \in U$.

The most usual SFN is $N_{S}(x)=1-x$. Additionally, let $N$ be a fuzzy negation on $U$ and $f: U^{n} \rightarrow U$ be a real function. The $N$-dual function of $f$ is given by :

$$
f_{N}\left(x_{1}, \ldots, x_{n}\right)=N\left(f\left(N\left(x_{1}\right), \ldots, N\left(x_{n}\right)\right)\right) .
$$

When $N$ is involutive, $\left(f_{N}\right)_{N}=f$, that is the $N$-dual function of $f_{N}$ coincides with $f$. In addition, if $f=f_{N}$ then it is clear that $f$ is a self-dual function [4].

\subsection{Aggregation Operators}

In [13, Definition 2], an $n$-ary aggregation is a function $A: U^{n} \rightarrow U$ demanding, for all $\vec{x}=\left(x_{1}, x_{2}, \ldots, x_{n}\right)$, $\vec{y}=\left(y_{1}, y_{2}, \ldots, y_{n}\right) \in U^{n}$, the following conditions:

A1: $A(\overrightarrow{0})=A(0,0, \ldots, 0)=0$ and $A(\overrightarrow{1})=A(1,1, \ldots, 1)=1$;

A2: If $\vec{x} \leq \vec{y}$ then $A(\vec{x}) \leq A(\vec{y})$;

A3: $A\left(\overrightarrow{x_{\sigma}}\right)=A\left(x_{\sigma_{1}}, x_{\sigma_{2}}, \ldots, x_{\sigma_{n}}\right)=A(\vec{x})$ when $\sigma: \mathbb{N}^{n} \rightarrow \mathbb{N}^{n}$ is a $\sigma$-permutation.

Some extra usual properties for aggregation functions are the following:

A3: Symmetry $A\left(\overrightarrow{x_{\sigma}}\right)=A\left(x_{\sigma_{1}}, \ldots, x_{\sigma_{n}}\right)=A(\vec{x})$, for all $\sigma$-permutation;

A4: Compensation $\min _{i=1}^{n}\left(x_{i}\right) \leq A(\vec{x}) \leq \max _{i=1}^{n}\left(x_{i}\right)$;

A5: Idempotency $A(x, x, \ldots, x)=x$, for all $x \in U$;

Proposition 1 Let $\sigma: \mathbb{N}_{n} \rightarrow \mathbb{N}_{n}$, with $\mathbb{N}_{n}=\{1, \ldots, n\}$, be a permutation ordering the elements: $x_{\sigma(1)} \leq \ldots \leq x_{\sigma(n)}$. Let $w_{1}, \ldots, w_{n}$ be non negative weights $\left(w_{i} \geq 0\right)$ such that $\sum_{i=1}^{n} w_{i}=1$. The operator $\mathcal{A}: U^{n} \rightarrow U$ which is named ordered weighted average aggregation operator (OWA-operator) and given as

$$
\mathcal{A}(\vec{x})=\sum_{i=1}^{n} w_{i} x_{\sigma(i)},
$$

verifies property $\mathbf{A} \mathbf{k}$, for $\mathbf{k} \in\{3,4,5\}$.

Proof: Straightforward.

Proposition 2 Let $\sigma: \mathbb{N}_{n} \rightarrow \mathbb{N}_{n}$ be a $\sigma$-permutation and $w_{1}, \ldots, w_{n}$ be non negative weights $\left(w_{i} \geq 0\right)$ such that $\sum_{i=1}^{n} w_{i}=1$. Then, $0 \leq \sum_{i=1}^{n} \frac{w_{i}}{\sigma(i)} \leq 1$.

Proof: For all $x_{\sigma(i)} \in U$, it is immediate that $1=\sum_{i=1}^{n} w_{i} \geq \sum_{i=1}^{n} \frac{w_{i}}{\sigma(i)} \geq 0$. 


\subsection{Triangular Norms and Conorms}

According with [6], a triangular sub(co)norm (t-sub(co)norm) is a binary aggregation function $T(S): U^{2} \rightarrow$ $U$ such that, for all $x, y, z \in U$, the following holds:
T0: $T(x, y) \leq \min (x, y)$;
S0: $S(x, y) \geq \max (x, y)$

and also verifying the commutativity, associativity and monotonicity properties which are, respectively, given by the next three expressions:

T1: $T(x, y)=T(y, x) ; \quad$ S1: $S(x, y)=S(y, x)$;

T2: $T(x, T(y, z))=T(T(x, y), z) ; \quad$ S2: $S(x, S(y, z))=S(S(x, y), z)$;

T3: $T(x, z) \leq T(y, z)$, if $x \leq y ; \quad$ S3: $S(x, z) \leq S(y, z)$, if $x \leq y$.

Additionally, a triangular (co)norm (t-(co)norm) is -sub(co)norm also satisfying the property:
T4: $T(x, 1)=x$;
S4: $S(x, 0)=x$.

Proposition 3 For $i \geq 1$ and $x, y \in U$, a function $T_{i}\left(S_{i}\right): U^{2} \rightarrow U$ is a $t$-sub(co)norm given by

$$
T_{i}(x, y)=\frac{1}{i} x y \quad\left(S_{i}(x, y)=1-\frac{1}{i}(1-x-y+x y)\right) .
$$

Proof: Straightforward.

When $i=1$, by Eq.(3)a and Eq.(3)b we obtain the product t-norm and probabilistic sum, which are mutual $N_{S}$-dual t-(co)norms, respectively given by

$$
T_{P}(x, y)=x y \quad\left(S_{P}(x, y)=x+y-x y\right) .
$$

\section{Fuzzy $e$-Xor and Dual Construction}

This section is based on the $\mathrm{X}(\mathrm{N})$ or connective concepts as described in [2].

Definition 1 A function $\mathrm{E}(\mathrm{D}): U^{2} \rightarrow U$ is a fuzzy subX(N)or if it satisfies, for all $x, y \in U$, the following properties:

E0(i): $\mathrm{E}(1,0)=1$;

E1: $\mathrm{E}(x, y)=\mathrm{E}(y, x)$;
DO(i): $\mathrm{D}(0,1)=0$;

D1: $\mathrm{D}(x, y)=\mathrm{D}(y, x)$;

E2(i): If $x \leq y$ then $\mathrm{E}(0, x) \leq \mathrm{E}(0, y) ; \quad$ D2(i): If $x \leq y$ then $\mathrm{D}(0, x) \geq \mathrm{D}(0, y)$;

E2(ii): If $x \leq y$ then $\mathrm{E}(1, x) \geq \mathrm{E}(1, y) ; \quad$ D2(ii): If $x \leq y$ then $\mathrm{D}(1, x) \leq \mathrm{D}(y, 1)$.

Definition 2 A fuzzy subX(N)or satisfying:

E0(ii): $\mathrm{E}(1,1)=\mathrm{E}(0,0)=0 ;(D O(i i): \mathrm{D}(1,1)=\mathrm{D}(0,0)=1)$

is called a fuzzy exclusive or (Xor) (fuzzy exclusive nor (XNor)).

Some extra reasonable properties can be considered for the fuzzy Xor connective (see [2] for a more thorough discussion):

E3: $\mathrm{E}(x, x) \neq 1$;

E4: If $\mathrm{E}(x, y)=1$ then $(x=1$ and $y=0)$ or $(x=0$ and $y=1)$. 
By considering any fuzzy negation, another generalization of this fuzzy Xor $\mathrm{E}_{K}$ is introduced in [1] and reported below. In [1, Prop. 3.6], when $T, S$ and $N$ are a t-norm, a t-conorm and a fuzzy negation, respectively, for all $x, y \in U$, the function $\mathrm{E}_{S, T}\left(\mathrm{D}_{T, S}\right): U^{2} \rightarrow U$, defined by

$$
\mathrm{E}_{S, T}(x, y)=S(x, y)-T(x, y) \quad\left(\mathrm{D}_{T, S}(x, y)=N(N(T(x, y))-N(S(x, y)))\right.
$$

is a fuzzy Xor (XNor) connective called in this paper as fuzzy e-X(N)or connective. Moreover, by [1, Prop. 3.7], it holds that $N_{\mathrm{E}_{S, T}}(x)=\mathrm{E}_{S, T}(1, x)=N(x)$.

Proposition 4 [1, Prop. 2] When $T=T_{P}, S=S_{P}$ and $N=N_{S}$ then, for all $x, y \in U$, it holds that

$$
\mathrm{E}_{S_{P}, T_{P}}(x, y)=x+y-2 x y \quad\left(\mathrm{D}_{T_{P}, S_{P}}(x, y)=1-x-y+2 x y\right), \quad \forall x, y \in U .
$$

Proposition 5 [1, Prop. 3] The fuzzy e-Xor connective $\mathrm{E}_{S_{P}, T_{P}}$ satisfies $\boldsymbol{E 3}$ and $\mathbf{E 4}$.

\subsection{Fuzzy e-X(N)or (co)implication class}

A fuzzy subimplicator $I: U^{2} \rightarrow U$ satisfies the conditions:

I0 : $I(1,1)=I(0,1)=I(0,0)=1$

A fuzzy subimplicator $I: U^{2} \rightarrow U$ also satisfying the boundary condition:

I1 : $I(1,0)=0$;

is called fuzzy implicator. And, a fuzzy (sub)implicator $I$ verifying:

I2: If $x \leq z$ then $I(x, y) \geq I(z, y)$ (left antitonicity);

I3 : If $y \leq z$ then $I(x, y) \leq I(x, z)$ (right isotonicity);

I4 : $I(0, y)=1$ (left boundary property);

is called a fuzzy (sub)implication [?, Def. 6][5].

There exist many classes of (co)implication functions (see, e.g., [3] and [?]). We consider in this paper the e-Xor (eXNor-coimplication) is studied [2], based on the equivalence of the classical logic: $\alpha \rightarrow \beta \equiv \neg \alpha \vee(\neg \alpha \oplus \beta)$.

Let $T(S), N$ and $\mathrm{E}(\mathrm{D})$ be a t-(co)norm, a fuzzy negation and a fuzzy Xor (XNor), respectively. According to [2], the function $I_{S, N, E}\left(J_{D, T, N}\right): U^{2} \rightarrow U$, given by

$$
I_{E, S, N}(x, y)=E(x, S(N(x), N(y))), \quad\left(J_{D, T, N}(x, y)=D(x, T(N(x), N(y)))\right) .
$$

is a fuzzy (co)implication, called $e$-Xor implication ( $e$-XNor coimplication). Moreover, $N_{I}: U^{2} \rightarrow U$, $N_{I}(x)=I(x, 0)$ is the fuzzy negation underlying $I$.

Proposition 6 When $S=S_{P}, N=N_{S}$ and $T=T_{P}, \forall x, y \in U$, then

$$
I_{E_{S_{P}, T_{P}}, S_{P}, N_{S}}(x, y)=1-x-x y+2 x^{2} y\left(J_{D_{T_{P}, S_{P}}, T_{P}, N_{S}}(x, y)=y-2 x+x y+2 x^{2}-2 x^{2} y\right)
$$

satisfies I $(\boldsymbol{J k})$ for $k \in\{1,2,3\}$.

\section{Aggregating Fuzzy Xor Conectives}

Definition 3 [12, Prop. 5.1] The function $\mathcal{F}_{A}: U^{k} \rightarrow U$ called as $(A, \mathcal{F})$-operator on $U$ is given by:

$$
\mathcal{F}_{A}\left(x_{1}, \ldots, x_{k}\right)=A\left(F_{1}\left(x_{1}, \ldots, x_{k}\right), \ldots, F_{n}\left(x_{1}, \ldots, x_{k}\right)\right) .
$$




\subsection{Aggregating fuzzy t-subnorms and t-subconorms}

Proposition 7 [12, Proposition 6.1] Let $A$ be an n-ary aggregation operator and $\left\{T_{1}, T_{2}, \ldots T_{n}\right\}$ and $\left\{S_{1}, S_{2}, \ldots, S_{n}\right\}$ be finite sets of $t$-subconomrs and $t$-subnorms both verifying distributivity and generalized associativity, respectively. The functions $\mathcal{T}_{A}, \mathcal{S}_{A}: U^{k} \rightarrow U$ called as $(A, \mathcal{T})$ - and $(A, \mathcal{S})$ - operators on $U$ and given by Eq.(9) are fuzzy $t$-subnorm and fuzzy $t$-subconorm, respectively.

\subsection{Aggregating Fuzzy $e-\mathrm{X}(\mathrm{N})$ or Connectives}

In this section, focusing on the fuzzy $e$-XNor class, a methodology is presented in order to obtain a new fuzzy $e$-subX(N)or connective from the OWA-operator and a finite subset of fuzzy $e$-subX(N)or connectives.

Proposition 8 The function $\mathrm{E}_{i}\left(\mathrm{D}_{i}\right): U^{2} \rightarrow U$ given by:

$$
\mathrm{E}_{i}(x, y)=1-\frac{1}{i}(1-x-y+2 x y)\left(\mathrm{D}_{i}(x, y)=\frac{1}{i}(1-x-y+2 x y)\right), \forall x, y \in U,
$$

whenever $i$ is a rational index such that $i \geq 1$, verifies $\mathbf{E k}(\mathbf{D k})$ for $\mathbf{k} \in\{0(i), 1,2(i), 2(i i), 3,4,5\}$.

Corollary 1 The function $\mathrm{E}_{i}\left(\mathrm{D}_{i}\right): U^{2} \rightarrow U$ in Eq. (10)a (Eq. (10)b) is a fuzzy e-subX(N)or connective.

In addition, the family of $e$-subX(N)or connectives $\mathrm{E}_{i}\left(\mathrm{D}_{i}\right)$ is referred as $\mathcal{E}(\mathcal{D})$.

Proposition 9 Let $\mathcal{A}: U^{n} \rightarrow U$ be an OWA-operator. For all $x, y \in U$, the function $\mathcal{E}_{\mathcal{A}}\left(\mathcal{D}_{\mathcal{A}}\right): U^{2} \rightarrow U$, called $(\mathcal{A}, \mathcal{E})$-operator $((\mathcal{A}, \mathcal{D})$-operator $)$ is given by

$$
\mathcal{E}_{\mathcal{A}}(x, y)=1-\sum_{i=1}^{n} \frac{w_{i}}{\sigma(i)}(1-x-y-2 x y) \quad\left(\mathcal{D}_{\mathcal{A}}(x, y)=\sum_{i=1}^{n} \frac{w_{i}}{\sigma(i)}(1-x-y-2 x y)\right) .
$$

Proof:For all $x, y \in U$. it holds that:

$$
\begin{aligned}
\mathcal{E}_{\mathcal{A}}(x, y) & =\mathcal{A}\left(E_{1}(x, y), \ldots,\left(E_{n}(x, y)\right)=w_{1} E_{\sigma(1)}(x, y)+\ldots+w_{n} E_{\sigma(n)}(x, y)\right. \\
& =\sum_{i=1}^{n} w_{i}\left(1-\frac{1}{\sigma(i)}(1-x-y+2 x y)\right)=\sum_{i=1}^{n} w_{i}-\sum_{i=1}^{n}\left(\frac{w_{i}}{\sigma(i)}(1-x-y+2 x y)\right) \\
& =1-\sum_{i=1}^{n} \frac{w_{i}}{\sigma(i)}(1-x-y-2 x y) .
\end{aligned}
$$

Therefore Eq.(13) is verified. The other one can be analogously done.

Proposition 10 Let $\mathcal{A}: U^{n} \rightarrow U$ be an OWA-operator. For all $x, y \in U$, the $(\mathcal{A}, \mathcal{E})$-operator $((\mathcal{A}, \mathcal{D})$ operator) verifies $\mathbf{E k}$ (Dk) for $\mathbf{k} \in\{0(i), 1,2(i), 2(i i), 3,4\}$.

Proof: For all $x, y \in U$ and $i \geq 1$ the following holds.

E0(i) Since $\mathcal{E}_{\mathcal{A}}(1,0)=1-\sum_{i=1}^{n} \frac{w_{i}}{\sigma(i)}(1-1-0-2 \cdot 0 \cdot 1)$ then $\mathcal{E}_{\mathcal{A}}$ verifies $E 0(i)$.

E1 By Eq. (10), the following holds: $\mathcal{E}_{\mathcal{A}}(x, y)=1-\sum_{i=1}^{n} \frac{w_{i}}{\sigma(i)}(1-x-y-2 x y)=1-\sum_{i=1}^{n} \frac{w_{i}}{\sigma(i)}(1-$ $y-x-2 y x)=1-\sum_{i=1}^{n} \frac{w_{i}}{\sigma(i)}(1-x-y-2 x y)$. Therefore, $\mathcal{E}_{\mathcal{A}}$ verifies $E 1$.

E2(i) By Prop.2, if $y_{1} \leq y_{2}$ then $\sum_{i=1}^{n} \frac{w_{i}}{\sigma(i)}\left(1-y_{1}\right) \geq \sum_{i=1}^{n} \frac{w_{i}}{\sigma(i)}\left(1-y_{2}\right)$ which means that $1-$ $\sum_{i=1}^{n} \frac{1}{i}\left(1-y_{1}\right) \leq \sum_{i=1}^{n} 1-\frac{1}{i}\left(1-y_{2}\right)$. Therefore, $\mathcal{E}_{\mathcal{A}}$ verifies $E 2(i)$.

E2(ii) Analogous to E2(i). 
E3: Since $1-2 x+x^{2}=0$ does not have rational zeros on $U$, we conclude that $\mathrm{E}(x, x) \neq 1$.

E4: When $\mathcal{E}_{\mathcal{A}}(x, y)=1$ we have that $\sum_{i=1}^{n} \frac{w_{i}}{\sigma(i)}(1-x-y-2 x y)=0$. Then, it implies that $1-x-$ $y-2 x y=0$ or $x=\frac{1-y}{1-2 y}$ Thus, we have as the possible solutions $(x=0$ and $y=1)$ or $(x=1$ and $y=0)$.

Analogously, it can be proved to the dual construction. Therefore Prop.10 is verified.

Corollary 2 Let $\mathcal{A}: U^{n} \rightarrow U$ be an OWA-operator. For all $x, y \in U$, the $(\mathcal{A}, \mathcal{E})$-operator $((\mathcal{A}, \mathcal{D})$ operator) is e-subX(N)or connective.

Proof:Straightforward from Prop.10.

\subsection{Aggregating Fuzzy $e-\mathbf{X}(\mathbf{N})$ or (co)implications}

In the following, focusing on the fuzzy $e$-XNor sub(co)implication class, a fuzzy sub(co)implication is obtained from the OWA-operator and a finite subset of fuzzy $e$-subX(N)or sub(co)implications.

Proposition 11 The function $I_{i}\left(\mathrm{D}_{i}\right): U^{2} \rightarrow U$ given by:

$$
I_{i}(x, y)=1-\frac{1}{i}\left(x-x y+2 x^{2} y\right)\left(J_{i}(x, y)=\frac{1}{i}(1-x)(2-y-2(1-x)(1-y))\right), \forall x, y \in U
$$

whenever $i$ is a rational index such that $i \geq 1$, verifies $\mathbf{I} \mathbf{k}(\mathbf{J k})$ for $\mathbf{k} \in\{0(i), 1,2(i), 2(i i), 3,4,5\}$.

Corollary 3 The function $I_{i}\left(J_{i}\right): U^{2} \rightarrow U$ in Eq.(12)a (Eq. (12)b) is a fuzzy e-subX(N)or (co)implication.

In addition, the family of $e$-subX(N)or connectives $I_{i}\left(J_{i}\right)$ is referred as $\mathcal{I}(\mathcal{J})$.

Proposition 12 Let $\mathcal{A}: U^{n} \rightarrow U$ be the OWA-operator. The function $\mathcal{E}_{\mathcal{A}}\left(\mathcal{D}_{\mathcal{A}}\right): U^{2} \rightarrow U$, called $(\mathcal{A}, \mathcal{E})$-operator $((\mathcal{A}, \mathcal{D})$-operator $)$ is given by

$\mathcal{I}_{\mathcal{A}}(x, y)=1-\sum_{i=1}^{n} \frac{w_{i}}{\sigma(i)}\left(x+x y-2 x^{2} y\right)\left(\mathcal{J}_{\mathcal{A}}(x, y)=\sum_{i=1}^{n} \frac{w_{i}}{\sigma(i)}(1-x)(2-y-2(1-x)(1-y))\right.$.

Proof:For all $x, y \in U$. it holds that:

$$
\begin{aligned}
\mathcal{I}_{\mathcal{A}}(x, y) & =\mathcal{A}\left(I_{1}(x, y), \ldots,\left(I_{n}(x, y)\right)=w_{1} I_{\sigma(1)}(x, y)+\ldots+w_{n} I_{\sigma(n)}(x, y)\right. \\
& =\sum_{i=1}^{n} w_{i}\left(1-\frac{1}{\sigma(i)}\left(x-x y+2 x^{2} y\right)\right)=\sum_{i=1}^{n} w_{i}-\sum_{i=1}^{n}\left(\frac{w_{i}}{\sigma(i)}\left(x-x y+2 x^{2} y\right)\right) \\
& =1-\sum_{i=1}^{n} \frac{w_{i}}{\sigma(i)}\left(x-x y+2 x^{2} y\right) .
\end{aligned}
$$

Therefore Eq.(13) is verified. The other one can be analogously done.

Proposition 13 Let $\mathcal{A}: U^{n} \rightarrow U$ be an OWA-operator. For all $x, y \in U$, the $(\mathcal{A}, \mathcal{I})$-operator $((\mathcal{A}, \mathcal{J})$ operator) verifies $\mathbf{E k}(\mathbf{D k})$ for $\mathbf{k} \in\{0(i), 1,2(i), 2(i i), 3,4\}$.

Proof: Analogous of Prop.10.

Corollary 4 Let $\mathcal{A}: U^{n} \rightarrow U$ be an OWA-operator. For all $x, y \in U$, the $(\mathcal{A}, \mathcal{E})$-operator $((\mathcal{A}, \mathcal{D})$ operator) is e-subX(N)or connective.

Proof: It follows from Prop.13. 


\section{Conclusion}

The study of aggregating fuzzy $e-\mathrm{X}(\mathrm{N})$ or connectives, their dual construction and corresponding fuzzy implications is main contribution of this work. Taking the class of strong fuzzy negation (standard negation), the probablistic sum and the product t-norm the paper formally states that the OWA-operator preserves the fuzzy $e-\mathrm{X}(\mathrm{n})$ or connectives and corresponding fuzzy $e-\mathrm{X}(\mathrm{N})$ or $(\mathrm{co})$ implications.

Based on the previous study of fundamental properties of other fuzzy X(N)or classes (see, e.g. [2, 7]), our current investigation aims clearly to contemplate other aggregation fuzzy $\mathrm{X}(\mathrm{N})$ or connectives also including their conjugate operators obtained by action of an automorphisms on $U$.

\section{References}

[1] B. Bedregal and R. Reiser and G. Dimuro, Xor-implications and E-implications: classes of fuzzy implications based on fuzzy Xor, Electronic Notes on Theoretical Computer Science, 247 (2009) 5-18.

[2] B. Bedregal and R. Reiser and G. Dimuro, Revising XOR-implications: Classes of Fuzzy (Co)implications based on Fuzzy XOR (XNOR) connectives, International Journal of Uncertainty, Fuzziness and Knowlegde- Based Systems, 14(6) (2013) 1-29.

[3] D. Dubois, W. Ostasiewisz and H. Prade, Fundamentals of Fuzzy Sets, Fuzzy Sets: History and Basic Notions, "The Handbook of Fuzzy Sets Series' ( Didier Dubois and Henri Prade, eds.), pp. 21106 Kluwer, Boston, 2000.

[4] J. Fodor and M. Roubens, "Fuzzy Preference Modelling and Multicriteria Decision Support", Dordrecht: Kluwer Academic Publisher, 1994.

[5] L. Kitainik, "Fuzzy Decision Procedures with Binary Relations", Dordrecht: Kluwer Academic Publisher, 1993.

[6] Klement, E.P., and Mesiar, R., and Pap, E. (2000). Triangular Norms. Dordrechet: Kluwer Academic Publisher.

[7] A. Lemke and R. Reiser and A. Du Bois and M. Pilla, Aggregation Operators on the Fuzzy e-Xor and e-XNor Classes, "WEIT 20133 - Proceedings of WEIT 2013" (IEEExplore) pp. 1-7, Pelotas, 2013.

[8] X. He, Y. Li and K. Qin, Fuzzy XNOR connectives in fuzzy logic, Soft Computing 15 (2011) 2457-2465.

[9] W. Pedrycz and G. Succi, fXOR fuzzy logic networks Soft Computing 7 (2002) 115-120 (DOI 10.1007/s00500-002-0179-5).

[10] A. Pradera, G. Beliakov, H. Bustince, Aggregation functions and contradictory information, Fuzzy Sets and Systems 191 (2012) 41-61.

[11] A. Pradera, A Review of the Relationships between Aggregation, Implication and Negation Functions, Aggregation Functions in Theory and in Practice - Advances in Intelligent Systems and Computing, 28 (2013) 31-36.

[12] R. Reiser and B. Bedregal and M. Baczyński, Aggregating Fuzzy Implications, Information Science. 253 (2013), 126-146.

[13] H. Bustince and T. Calvo and B. Baets and J.Fodor and R. Mesiar and J. Montero and D. Paternain and A. Pradera" A class of aggregation functions encompassing two-dimensional OWA operators, Information Science, 180(10), (2010) 1977-1989. 